\title{
A single-cell atlas of human fetal lung development between 14 and 19 weeks of gestation
}

Laurent Renesme ${ }^{1,2,3}$, Flore Lesage ${ }^{3}$, David Cook $^{4}$, Shumei Zhong ${ }^{3}$, Satu Hänninen ${ }^{5}$, Olli Carpén ${ }^{5}$, Ivana Mižíková*6 ${ }^{*}$ Bernard Thébaud ${ }^{\star 3,7,8}$.

\footnotetext{
${ }^{1}$ Neonatal Intensive Care Unit, University Hospital of Bordeaux, France

${ }^{2}$ INSERM, Centre de Recherche Cardio-Thoracique de Bordeaux, U1045, F-33600 Pessac, France

${ }^{3}$ Sinclair Center for Regenerative Medicine, Ottawa Hospital Research Institute, Ottawa, ON, Canada

${ }^{4}$ Lunenfeld-Tanenbaum Research Institute, Toronto, Ontario, Canada

${ }^{5}$ Precision Cancer Pathology, department of Pathology and research Program in System Oncology, University of Helsinki and HUS Diagnostic Center, Helsinki University Hospital, Helsinki, Finland

${ }^{6}$ Experimental Pulmonology, Department of Pediatrics and Adolescent Medicine, Faculty of Medicine and University Hospital Cologne, University of Cologne, Cologne, Germany

${ }^{7}$ Department of Cellular and Molecular Medicine, University of Ottawa, Ottawa, ON, Canada

${ }^{8}$ Neonatology, Department of Pediatrics, Children's Hospital of Eastern Ontario (CHEO) and CHEO Research Institute, Ottawa, ON, Canada

"These authors contributed equally: Ivana Mižíková and Bernard Thébaud
}

Corresponding author: Dr. Bernard Thébaud

\author{
Senior Scientist, Ottawa Hospital Research Institute; \\ Professor of Pediatrics, University of Ottawa; \\ Staff Neonatologist at The Ottawa Hospital and CHEO \\ 401 Smyth Road/501 Smyth Road \\ Ottawa, Ontario \\ Phone: 613-737-8899 ext: 73905 \\ Email: bthebaud@toh.ca
}

\section{TAKE HOME MESSAGE}

Using a single-cell transcriptomic approach (single-nuclei RNA sequencing), we describe here, for the first time, the cellular landscape, cell developmental trajectories, and cell-to-cell communication in the developing human lung during the pseudoglandular stage. 


\section{ABSTRACT}

Rationale. Human lung development has been mainly described in morphologic studies and the potential underlying molecular mechanisms were extrapolated from animal models. Therefore, there is a need to gather knowledge from native human lung tissue. In this study we describe changes at a single-cell level in human fetal lungs during the pseudoglandular stage.

Methods. We report the cellular composition, cell trajectories and cell-to-cell communication in developing human lungs with single-nuclei RNA sequencing (snRNA-seq) on 23,251 nuclei isolated from nine human fetuses with gestational ages between 14 to 19 weeks of gestation.

Results. We identified nine different cell types, including a rare pulmonary neuroendocrine cells population. For each cell type, marker genes are reported, and selected marker genes are used for spatial validation with fluorescent RNA in situ hybridization. Enrichment and developmental trajectory analysis provide insight into molecular mechanisms and signaling pathways within individual cell clusters according to gestational age. Lastly, ligand-receptor analysis highlights determinants of cell-to-cell communication among the different cell types through the pseudoglandular stage, including general developmental pathways (NOTCH and TGFB), as well as more specific pathways involved in vasculogenesis, neurogenesis, and immune system regulation.

Conclusion. These findings provide a clinically relevant background for research hypotheses generation in projects studying normal or impaired lung development and help to develop and validate surrogate models to study human lung development, such as human lung organoids. 


\section{INTRODUCTION}

Human lung development is traditionally divided into 5 stages: embryonic (1-7 weeks of gestation (WG)), pseudoglandular (5-17 WG), canalicular (16-26 WG), saccular (24 to 38 WG), and alveolar (36 WG to 3 years, with late alveolarization continuing up to early adulthood $)^{1}$. While these stages have been defined morphologically and histologically during human fetal development, very little is known about the underlying molecular and cellular mechanisms ${ }^{2,3}$. Detailed insight into the molecular pathways and cellular communication which contribute to lung development would improve our understanding of lung repair as it may recapitulate fetal lung development, and would enable us to develop new therapeutic targets ${ }^{4,5}$. Due to developmental similarities in human and rodent lungs, mouse and rats have been used extensively to study lung development ${ }^{6}$. Airway branching morphogenesis in rodents is well described, and gain- and loss-of-function studies using genetically modified animals have identified the critical factors of this developmental process ${ }^{3,7}$. However, some compartments of the lung, such as stroma or vascular and neuronal networks, remain poorly explored during the fetal developmental period despite their potential key roles in lung development ${ }^{8,9}$. While versatile in experimental settings, rodent models of lung development have several limitations, including species-specific maturation and function at birth, and molecular regulation ${ }^{10,11}$. Therefore, there is a need to unravel cellular composition, developmental trajectories, and signaling within the developing human lung.

Single-cell and single-nuclei RNA sequencing (scRNA-seq and snRNA-seq, respectively) can reveal complex and rare populations, uncover regulatory relationships, and track distinct cell lineages in development. In addition, computational methods have been developed to analyze cellular communications and to determine which extracellular signals influence the cell population of interest.

In the present study, we report the cellular composition, developmental pathways and cell-to-cell communication in fetal human developing lungs using snRNA-seq approach on 23,251 nuclei isolated from nine lungs with gestational ages (GAs, described in WG) 
spanning through the pseudoglandular and canalicular stages (14 to $19 \mathrm{WG}$ ). We describe nine different cells populations, including rare cell population of pulmonary neuroendocrine cells (PNEC). For each identified population we report marker genes and enrichment and developmental state analysis. Additionally, we provide a spatial validation for selected populations using fluorescent RNA in situ hybridization (FISH). Lastly, ligand-receptor analysis highlights determinants of cell-to-cell communication among the different cell types. 


\section{METHODS}

A full description of the experimental procedures can be found in the supplementary methods.

\section{Human fetal tissue collection}

De-identified human fetal lung samples were obtained under REB approval by The Ottawa Hospital Review Ethical Board (20170603-01H). Lung tissue was snap frozen and stored at $-80^{\circ} \mathrm{C}$.

\section{Single-nuclei suspension preparation}

Single nuclei isolation was performed according to Martelotto et al. ${ }^{12}$ with minor adjustments. Sample multiplexing was performed using the MULTI-seq protocol as reported before $^{13,14}$. Nuclei integrity was confirmed by fluorescent microscopy (Axio Imager M2, Carl Zeiss, Toronto, ON, Canada) and single diploid nuclei were sorted using a flow cytometer (BD LSR Fortessa, Beckton Dickinson Biosciences, Franklin Lakes, NJ, USA) (Supplemental figure 1a-b)

\section{Single-nuclei sequencing}

Cell capture and library production was performed with the Chromium system (10X Genomics, Pleasanton, CA, USA). Sequencing was performed with NextSeq500 (Illumina, San Diego, CA, USA). Raw sequencing reads were processed using CellRanger v3.1.0. All analyses were performed with Seurat v4.0.0 ${ }^{15}$. SCTransform() was used to normalize samples, select highly variable genes, and to regress out cell cycle and cell stress effects. To eliminate batch effects or biological variability effects on clustering, the data integration method implemented by Seurat for SCTransform-normalized data was performed, using the SelectIntegrationFeatures(), PrepSCTIntegration(), FindIntegrationAnchors(), and 
IntegrateData() functions. Differential State Analysis (DSA) was performed using muscat $\mathrm{R}$ package $^{16}$. Gene set enrichment analysis (GSEA) was performed with Metascape ${ }^{17}$. Cell communications were inferred using NicheNet (v1.0.0) R package ${ }^{18}$.

\section{In situ hybridization}

FISH was performed on formalin-fixed, paraffin embedded human fetal lungs. $3 \mu \mathrm{m}$ thick sections were analysed using the RNAscope technology following the manufacturer's protocol, and as previously described ${ }^{14}$. 


\section{RESULTS}

\section{Cellular landscape of the human developing lung}

To characterize the cellular composition in developing human lungs, we generated snRNA-seq profiles of 23,251 nuclei isolated from nine fetal lung samples with GAs between 14+1 and 19+0 weeks (Figure 1a, and Supplemental figure 1c). We identified nine distinct cell populations, present across all GAs (Figure 1b-d), and described the lung cellular composition variation over time. While proportions of both stromal populations decreased over time ( 83.7 and $6.1 \%$ at $14+1$ weeks vs. 47.6 and $4 \%$ at $19+0$ weeks), the size of distal airway epithelium cluster increased (from 3.6\% to 39.5\%) (Figure 1c and Supplemental table 1). Each cell cluster displayed a unique molecular signature characterized by multiple DEGs (Figure 1e and Supplemental table 2), and cell populations were annotated based on the expression of canonical cell lineage markers (Figure 1f and 2a). Additionally, among the top ten DEGs in some clusters, we identified less commonly used transcriptional patterns (Figure 1e). For example, pericytes expressed genes involved in the cGMP-PKG pathway such as MEF2C, PDE3A, TRPC6, and PLCB. Vascular and lymphatic endothelium expressed genes involved in angiogenesis, respectively EPAS1, PTPRB, CALCRL and NRP2, VAV3, STAB2. Finally, the immune cells cluster expressed HDAC9, SAMSN1 and DOCK8 that are involved in lymphocyte activation.

To further confirm clusters identities, and to gain further insights into the underlying molecular functions and biological processes, we performed an GSEA. Cell-type specific terms were associated with all the identified clusters. As expected, stromal populations 1 and 2 were associated with general terms such as extracellular matrix and mesenchyme development. Distal airway epithelium was associated with respiratory gaseous exchange, as well as apical and basolateral plasma membrane terms, while ciliated cells were characterized by cilium-specific pathways. Blood vessel development was associated with both, pericytes and vascular endothelium, while lymph vessel development pathways were specific to lymphatic endothelium cluster. Immune cells cluster was characterized by 
interleukin signalling and myeloid cell differentiation pathways. Finally, transsynaptic signaling was associated specifically with pulmonary neuroendocrine cells (Figure $2 b$, Supplemental table 3).

\section{Developmental changes in gene expression in developing lung stromal cells}

Within the stromal population (stroma 1 and 2), we identified three subclusters with distinct gene expression patterns represented across different GAs (Figure 3a-e, Supplemental table 4). Stromal subclusters 0 and 1 expressed traditional matrix fibroblast markers (FN1, MEOX2) (Figure 3f). Pulmonary fibroblasts can be typically segregated into two subtypes based on the distinct expression of COL13A1 and COL $14 A 1^{19}$. In our dataset the expression of COL13A1 was most prominent in stromal subcluster 0 , which was also characterized by expression of additional genes associated with $\operatorname{COL} 13 A 1^{+}$fibroblasts (ITGA8, LIMCH1, MYLK, PLXDC2, MACF1) (Figure 3f). A sizeable fraction of subcluster 1 expressed COL14A1, as well as other matrix fibroblast markers (FBLN1, COL1A2, AKAP12, COL1A1) (Figure 3f). Stromal subcluster 2 showed similarities to mesenchymal progenitors as described by Xie et al. ${ }^{19}$ and the LungGENS database ${ }^{20}$, characterized by the expression of SDC2 and SMARCC1 (Figure 3f). However, this sub-population did not express any proliferative genes such as TOP2A or MKI67 which are typically expressed in self-renewing progenitor populations. GSEA of stromal subclusters revealed supportive roles for the stromal cells in lung and mesenchyme development, with subcluster 0 and 1 being associated with regulation of organ architecture (connective tissue development, cell junction assembly, cytoskeleton organization, negative regulation of locomotion/cell motility) and subcluster 2 being associated with proliferative/stemness functions (DNA binding transcription factor activity, negative regulation of cell differentiation) (Supplemental figure 2a and c, Supplemental table 5).

Next, we performed DSA-based GSEA (Supplemental tables 6, 7 and 8). In the two main stromal clusters, Stroma 1 cluster was associated with response to growth factor and 
MTORC1 signaling for early GA (14+1 to $14+3 \mathrm{WG})$, and regulation of cell communication by electrical coupling, regulation of vascular associated smooth muscle contraction for late GA $(18+2$ to $19+0$ WG) (Figure 3g, Supplemental figure 3). For stroma 2, prominent regulated enriched pathways included TNF $\alpha$ signaling via NFKB for early GA, as well as muscle structure development and Kit receptor signaling pathway for late GA (Figure 3h, Supplemental figure 3).

\section{Developmental changes in gene expression in developing airway epithelium}

Upon subclustering of the distal airway epithelium, we identified three subclusters with distinct gene expression represented across the different GAs (Figure 4a-e, Supplemental table 4). Epithelial subclusters shared common marker genes with bud tip progenitors (subcluster 0: SFTPC, ETV5), bud tip adjacent (subcluster 1: DMD, MECOM), and secretory progenitor (subcluster 2: SCGB3A2, CFTR) epithelial cells as identified by Miller et al. ${ }^{21}$ in human fetal lung tissue of similar GA (Figure 4f). In addition, a GSEA was performed with DEG from these three subclusters (Supplemental figure $2 b$ and $d$, Supplemental table 5). Based on DEG and DSA results, we selected gene markers for spatial validation on the fetal lung tissue by FISH (Supplemental tables 2 and 6). Due to their cluster specificity, we selected SEMA3C and SFTPB as potential early and late distal airway epithelium markers in fetal lung (Supplemental figure 4a). As seen by FISH (Figure 4g), distal airway epithelium (marked by $F G F R 2^{+}$) in earlier GAs co-expresses SEMA3C, with only few cells also co-expressing SFTPB. However, the majority of $F G F R 2^{+}$epithelial cells surrounding the airway lumen at later GAs were SEMA3C and SFTPB'. Notably, an adjacent layer of $F G F 2 / S E M A 3 C^{+} / S F T P B$ cells can also be seen (Figure $\left.4 \mathrm{~h}\right)$. Finally, based on the DSA, we performed a GSEA. Among the most regulated pathways in distal airway epithelium were sensory organ development and blood vessel development (Figure 4h, Supplemental figure 3, Supplemental tables 7 and 8). 
In the case of ciliated cells and PNECs, DSA identified only few DEGs, confirming previous reports on the early specification of these cell populations (Supplementary figures 5 and 6 , respectively) ${ }^{22,23}$. Interestingly, only a very small fraction of the PNEC population showed high expression of typical PNEC markers such as GRP1, CALCA, ASCL1, and $S Y P^{21}$, whereas markers associated with the synapse, signal release and transsynaptic signaling (NRXN1, PTPRN2, PCLO, CADPS) were expressed in the majority of the PNECs in our dataset. FISH was performed using NRXN1 and GRP as PNEC markers (Supplemental figure $4 \mathrm{~b}$ ). The $N R X N 1^{+} / G R P^{+}$PNECs were present at all GAs and were either adjacent to or localized within the airway epithelium. Both, isolated cells and small cells clusters (neuroendocrine bodies) were noted. Interestingly, while all $G R P^{+}$cells were $N R X N 1^{+}, G R P / N R X N 1^{+}$cells were also noted, suggesting a possible existence of two subpopulations of PNECs in the developing lung (Figure 5).

\section{Developmental changes in gene expression in developing endothelial, pericyte and immune cells populations}

Vascular endothelium at early GA (14+3 WG) was characterized by cell type specific pathways such as VEGFA-VEGFR2 signaling pathway, endothelium development and vasculature development (Figure 6a, Supplemental figure 3). In the lymphatic endothelium, genes expressed at early GAs were involved in ubiquitin protein ligase binding and IL-6 signaling. Interestingly, ubiquitination plays a central role in $\mathrm{NOTCH}$ signaling ${ }^{24}$ and IL-6 is involved in lymph angiogenesis via VEGF-C ${ }^{25}$, whereas later GAs were characterized by expression of genes involved in "cholesterol biosynthesis" (Supplemental figure 7). Next, to visualize the vascular endothelial cells in the fetal lung we performed a FISH (Figure 6b). Based on the DSA (Supplemental table 6), we selected KITLG as an early, and CAV1 as a late marker for vascular endothelium during the pseudoglandular phase (Supplemental figure 4c). However, both $\mathrm{KITLG}^{+} / \mathrm{PECAM}^{+}$and $\mathrm{CAV}^{+} / \mathrm{PECAM}^{+}$double positive cells could be found at all investigated GAs (Figure 6c). 
The pericytes cluster showed gene expression associated with regulation of vascular wound healing and cellular response to hormone stimulus at early GAs. In addition, cellular components involved in pericytes functions, such as filipodium and $Z$ disc were described at later GAs $(16+0 \text { and } 16+3 \text { WG, respectively })^{26,27}$ (Figure 6c). PDGFRB pericytes (Supplemental figure $4 d$ ) could be found in immediate proximity to vascular endothelium (defined by the expression of $P E C A M 1^{+}$) in the lungs throughout the pseudoglandular development (Figure 6d).

Finally, in the case of immune cells population, the DEGs at early GAs were associated with "lymphocyte activation" and "TNFa signaling via NFK $\beta$ " which is known to be crucial in lymphocyte activation, as well the specification of innate and adaptive immunity ${ }^{28}$ (Supplemental figure 8).

\section{Cellular crosstalk in the human developing lung}

In order to understand cell communication networks within the developing human fetal lung samples, we performed a ligand-receptor interaction analysis using $\mathrm{NicheNet}^{18}$. The analysis focused on time-dependent changes in cell communication rather than stable cell crosstalk, and the set of gene of interest used for the analysis was defined by differential expression between early GAs (14+1 and 14+3 WG, reference condition) and late GAs (18+0 and 19+0 WG, condition of interest). Cellular crosstalks identified at later GAs are presented in Figure 7, with the identified receptors and predicted target genes involved (Figure 8). GSEA on predicted target genes are presented in Supplemental table 9. Inferred cellular communications at later GAs were associated with general pathways such as NOTCH and TGFB pathways, as well as in pathways involved in immune system regulation, and neurogenic tissue and vasculature development.

We identified key factors in the NOTCH signaling pathway such as DLL1 (expressed by the ciliated cells) and its receptors NOTCH2 (expressed in stroma 1 and distal airway epithelium) and $\mathrm{NOTCH3}$ (distal airway epithelium). Predicted target genes were associated 
with T cell differentiation and TNF signaling in stroma 1 cluster, and with cellular senescence in airway epithelium. Moreover, DLL4 expressed by the vascular endothelium targeted changes in gene expression associated with INFY-mediated signaling and leukocytes cellcell adhesion in lymphatic endothelium via its receptor NOTCH1. ADAM17, an important NOTCH signaling activator, was expressed by majority of cell populations, while its receptor ITGB1 was expressed by distal airway epithelium. Potential ADAM17 target genes were primarily associated with inflammatory response.

TGFB1 was expressed by lymphatic and vascular endothelium and the immune cells. Its receptors, TGFBR1 and TGFBR2, were expressed in distal airway epithelium and the predicted target genes were associated with cellular senescence and gastrin signaling. $B M P 5$, another member of the TFGB family, was expressed in both stromal clusters, while its receptors (BMPR2, BMPR1A, ACVR2A and ACVR1) were specifically expressed by stroma 2. The targeted genes were associated with insulin resistance, T cell differentiation, IL-18 signaling, and with regulation of cell adhesion and motility.

CD44 was expressed in stroma 1 and immune cells and its potential ligands HAS2 and FN1 were expressed by stroma 1 and 2 clusters. Predicted target genes for CD44 activation in stroma 1 were associated with T cell differentiation and regulation of adaptative immune response. Target genes in immune cells cluster were associated with IL-18 and NFkappa B signaling pathways.

Further regulated were also genes involved in neurogenic tissue development, including stroma-expressed NTNG1, and its receptor LRRC4C, NEGR1 (expressed by ciliated cells and PNECs) and its receptor NEGR1 (stroma 1), as well as NCAM1 (stroma, lymphatic, and PNEC clusters) and its receptor ROBO1 (distal airway epithelium and vascular endothelium). TNC, an extracellular matrix protein implicated in the guidance of migrating neurons, was expressed in stroma 2 and distal airway epithelium clusters, with its receptors expressed in distal airway epithelium and vascular endothelium. 
Lastly, in regard to vasculature development, VEGF ligands were expressed by distal airway epithelium (VEGFA), vascular endothelium (VEGFC), and PNEC (VEGFB). VEGFA receptors were expressed in pericytes (NRP1, NRP2, FLT1) with predicted target genes associated with TGF-beta receptor signaling and positive regulation of cell migration, and in lymphatic endothelium (NRP1, NRP2, KDR) with predicted target genes associated with INFY-mediated signaling and regulation of leukocytes cell-cell adhesion. VEGFB receptor NRP1 was expressed in stroma 1, while NRP2 was expressed in immune cells, and the predicted target genes were associated with NF-kappa B signaling. In addition, we reported the expression of SEMA3E and its receptor PLXND1, which are required for normal vascular patterning during embryogenesis. SEMA3E was expressed by the airway epithelium (ciliated cells, PNEC and distal airway epithelium) and PLXND1, was expressed by the vascular endothelium. 


\section{DISCUSSION}

This is the first report of an unbiased transcriptomic analysis of human fetal lung samples, providing a detailed cell atlas of the human developing lung during pseudoglandular and early canalicular stages at a single-cell resolution. We identified 9 cell types, including rare cell type PNEC, and described developmental changes in gene expression and cell-cell communication across GAs. In addition to canonical marker genes, we identified an array of marker genes for each cell population and validated the spatial localization of specific cell populations such as PNEC and pericytes in the human developing lung. Lastly, the cell communication analysis provided unique insight into detailed cellular crosstalk and main pathways involved in the pseudoglandular stage of lung development.

Only few studies to date have reported the use of single-cell transcriptomic on human developing lung, typically including a small number of samples or focusing on specific cell types ${ }^{21,29,30}$. Our study reported transcriptomics data from 9 fetuses, representing each developmental week from 14 and 19 WG, allowing detailed analyses of gene expression changes overtime. Additionally, choosing single-nuclei isolation from frozen material, rather than single-cell isolation from fresh tissue, enabled us to isolate a relatively large number of distal airway epithelial cells (up to $39.5 \%$ of all cells at $19+0$ WG). This allowed us to show a steady increase in distal airway epithelial cells across GAs in relation to airway development, whereas previous studies using single-cell isolation approach seemed more prone to dissociation bias (with epithelial cells representing less than $4 \%$ of the total cells) ${ }^{29,30}$. These findings suggest that transcriptomic analysis of tissue with high stromal content, such as human fetal lungs, might be more efficient when using a single-nuclei, rather than a singlecell isolation preparation.

The main strength of this study lies within the detailed description of the cellular crosstalk changes between early $(14+1$ and $14+3$ WG) and late $(18+2$ and 19+0 WG) GAs. We identified several critical pathways associated with normal lung development and lung disease. For instance, VEGF plays a central role in lung development and maintenance, and 
is involved in the pathogenesis of many lung diseases, including emphysema, chronic obstructive pulmonary disease, bronchopulmonary dysplasia, pulmonary hypertension, acute lung injury, and asthma ${ }^{31}$. ADAM17, a NOTCH activator, plays a key role in lung inflammation regulation by increasing epithelium and smooth muscle cells permeability, secretion of inflammatory mediators, and trans-endothelial leukocyte migration, and its deficiency leads to decreased airway branching ${ }^{32}$. In addition, the role of the neurogenic tissue and vasculature in the developing lung is rapidly gaining interest ${ }^{8,9}$. Here we have identified key factors for neurogenic tissue development also known to be involved in abnormal lung development, such as $R O B O 1$ (expressed in distal airway epithelium and vascular endothelium) and TNC (expressed in stroma 2 and distal airway epithelium). Robo1 knock-out mice exhibit delayed lung maturation with increased mesenchymal cellularity and reduced terminal air spaces leading to respiratory distress and death at birth ${ }^{33}$. TNC inactivation in mice induces abnormal lung development and persistent abnormal lung function after birth ${ }^{34}$. Lastly, we described crosstalk between immune cells and stroma populations via CD44-HAS2. CD44 plays an important role in the regulation of lung fibroblasts senescence and apoptosis and the interaction HAS2-CD44 can induce pulmonary fibrosis via its receptor ${ }^{35}$. Immune cells population in our dataset were not well differentiated but additional data suggest that lung immune cells complexity and heterogeneity increase later during the gestation and after birth and play an important role in stroma remodelling and angiogenesis in the developing lung ${ }^{36}$.

Distal airway epithelial cells were among the most active signal-receiving cell types in our dataset, displaying active communication with all other cell populations including themselves. Our dataset further identifies a cluster of rare PNEC, and its interaction with both stroma 1 and 2, pericytes and vascular endothelium populations. The exact role of PNEC in lung development is yet to be described ${ }^{37}$. However, GRP expression had previously been detected in human fetal lung at 8 WG with peak of expression at midgestation. Moreover, elevated urine GRP was previously associated with the development of 
BPD suggesting an important role for PNECs in normal and impaired lung development ${ }^{38}$. In addition, FISH identified 2 populations of PNEC within the fetal lung tissue $\left(G R P^{+} / N R X N 1^{+}\right.$and $G R P / N R X N 1^{+}$cells). These findings suggest that $N R X N 1$ might be a more specific marker for PNEC than GRP.

Our study has several limitations. Firstly, the study doesn't consider the potential effects of fetus sex on the lung development whereas sex-related differences in human fetal lung transcriptome during the pseudoglandular stage had been previously reported ${ }^{39}$. However, the same study also found GA to have a more dominant effect on transcriptome than sex. Secondly, we assumed the fetuses were healthy as they were issued from selective abortions with no fetal indications. Nevertheless, all the fetuses had a GA below 20 weeks of gestation, prior to typical ultrasound morphological assessment for congenital malformation. Additional limitations relate to the downstream analysis. During development, important changes in cell differentiation and signalling can be induced by upregulation or downregulation in gene expression. While the presented DSA approach provides information on up-regulated genes across the different GA, it does not allow to study down-regulation in gene expression.

We report here, for the first time, an unbiased transcriptomic analysis of human fetal lungs during pseudoglandular stage at a single-cell resolution. This transcriptomic approach at a single cell level, combined with other novel approaches such as proteomics and metabolomics analysis ${ }^{40,41}$, are critical to unravel molecular pathways and cell communication in the human developing lung. Altogether, they will provide a clinically relevant background for the generation of novel research hypotheses in studies of normal or impaired lung development, help to develop and validate surrogate models to study human lung development and ultimately dentify new therapeutic targets. 


\section{REFERENCES}

1 Burri PH. Fetal and Postnatal Development of the Lung. Annu Rev Physiol 1984; 46: 617-628.

2 Schittny JC. Development of the lung. Cell Tissue Res 2017; 367: 427-444.

3 Morrisey EE, Hogan BLM. Preparing for the First Breath: Genetic and Cellular Mechanisms in Lung Development. Developmental Cell 2010; 18: 8-23.

$4 \quad$ Herriges M, Morrisey EE. Lung development: orchestrating the generation and regeneration of a complex organ. Development 2014; 141: 502-513.

$5 \quad$ Ubags NDJ, Alejandre Alcazar MA, Kallapur SG, Knapp S, Lanone S, Lloyd CM et al. Early origins of lung disease: towards an interdisciplinary approach. Eur Respir Rev 2020;

29. doi:10.1183/16000617.0191-2020.

$6 \quad$ Nardiello C, Mižíková I, Morty RE. Looking ahead: where to next for animal models of bronchopulmonary dysplasia? Cell Tissue Res 2017; 367: 457-468.

7 Cardoso WV, Lü J. Regulation of early lung morphogenesis: questions, facts and controversies. Development 2006; 133: 1611-1624.

8 Kina YP, Khadim A, Seeger W, El Agha E. The Lung Vasculature: A Driver or Passenger in Lung Branching Morphogenesis? Front Cell Dev Biol 2021; 8: 623868.

9 Bower DV, Lee H-K, Lansford R, Zinn K, Warburton D, Fraser SE et al. Airway branching has conserved needs for local parasympathetic innervation but not neurotransmission. BMC Biol 2014; 12: 92.

10 Danopoulos S, Shiosaki J, Al Alam D. FGF Signaling in Lung Development and Disease: Human Versus Mouse. Front Genet 2019; 10: 170.

11 Nikolić MZ, Caritg O, Jeng Q, Johnson J-A, Sun D, Howell KJ et al. Human embryonic lung epithelial tips are multipotent progenitors that can be expanded in vitro as long-term self-renewing organoids. eLife 2017; 6: e26575.

12 Martelotto L. 'Frankenstein' protocol for nuclei isolation from fresh and frozen tissue for snRNAseq. 2020. doi:10.17504/protocols.io.3fkgjkw.

13 McGinnis CS, Patterson DM, Winkler J, Conrad DN, Hein MY, Srivastava V et al. MULTI-seq: sample multiplexing for single-cell RNA sequencing using lipid-tagged indices. Nat Methods 2019; 16: 619-626.

14 Hurskainen M, Mižíková I, Cook DP, Andersson N, Cyr-Depauw C, Lesage F et al. Single cell transcriptomic analysis of murine lung development on hyperoxia-induced damage. Nat Commun 2021; 12: 1565.

15 Hao Y, Hao S, Andersen-Nissen E, Mauck WM, Zheng S, Butler A et al. Integrated analysis of multimodal single-cell data. Cell 2021; : S0092867421005833.

16 Crowell HL, Soneson C, Germain P-L, Calini D, Collin L, Raposo C et al. muscat detects subpopulation-specific state transitions from multi-sample multi-condition single-cell transcriptomics data. Nat Commun 2020; 11: 6077. 
17 Zhou Y, Zhou B, Pache L, Chang M, Khodabakhshi AH, Tanaseichuk O et al. Metascape provides a biologist-oriented resource for the analysis of systems-level datasets. Nat Commun 2019; 10: 1523.

18 Browaeys R, Saelens W, Saeys Y. NicheNet: modeling intercellular communication by linking ligands to target genes. Nat Methods 2020; 17: 159-162.

19 Xie T, Wang Y, Deng N, Huang G, Taghavifar F, Geng Y et al. Single-Cell Deconvolution of Fibroblast Heterogeneity in Mouse Pulmonary Fibrosis. Cell Rep 2018; 22: 3625-3640.

20 Du Y, Guo M, Whitsett JA, Xu Y. 'LungGENS': a web-based tool for mapping singlecell gene expression in the developing lung. Thorax 2015; 70: 1092-1094.

21 Miller AJ, Yu Q, Czerwinski M, Tsai Y-H, Conway RF, Wu A et al. In Vitro and In Vivo Development of the Human Airway at Single-Cell Resolution. Dev Cell 2020; 53: 117128.e6.

22 Cutz E, Gillan JE, Bryan AC. Neuroendocrine cells in the developing human lung: morphologic and functional considerations. Pediatr Pulmonol 1985; 1: S21-29.

23 Ernst LM, Ruchelli ED, Huff DS (eds.). Color Atlas of Fetal and Neonatal Histology. Springer New York: New York, NY, 2011 doi:10.1007/978-1-4614-0019-6.

24 Moretti J, Brou C. Ubiquitinations in the Notch Signaling Pathway. IJMS 2013; 14: 6359-6381.

25 Huang Y-H, Yang H-Y, Huang S-W, Ou G, Hsu Y-F, Hsu M-J. Interleukin-6 Induces Vascular Endothelial Growth Factor-C Expression via Src-FAK-STAT3 Signaling in Lymphatic Endothelial Cells. PLoS One 2016; 11: e0158839.

26 Hung CF, Wilson CL, Schnapp LM. Pericytes in the Lung. In: Birbrair A (ed). Pericyte Biology in Different Organs. Springer International Publishing: Cham, 2019, pp 41-58.

27 Yamazaki T, Mukouyama Y. Tissue Specific Origin, Development, and Pathological Perspectives of Pericytes. Front Cardiovasc Med 2018; 5: 78.

28 Bonizzi G, Karin M. The two NF-kappaB activation pathways and their role in innate and adaptive immunity. Trends Immunol 2004; 25: 280-288.

29 Danopoulos S, Bhattacharya S, Mariani TJ, Al Alam D. Transcriptional characterisation of human lung cells identifies novel mesenchymal lineage markers. Eur Respir J 2020; 55: 1900746.

30 Bhattacharya S, Myers JL, Baker C, Guo M, Danopoulos S, Myers JR et al. Single cell transcriptomic profiling identifies molecular phenotypes of newborn human lung cells. Genomics, 2020 doi:10.1101/2020.06.16.156042.

31 Voelkel NF, Vandivier RW, Tuder RM. Vascular endothelial growth factor in the lung. American Journal of Physiology-Lung Cellular and Molecular Physiology 2006; 290: L209L221.

32 Dreymueller D, Uhlig S, Ludwig A. ADAM-family metalloproteinases in lung inflammation: potential therapeutic targets. Am J Physiol Lung Cell Mol Physiol 2015; 308: L325-343.

33 Xian J, Clark KJ, Fordham R, Pannell R, Rabbitts TH, Rabbitts PH. Inadequate lung 
development and bronchial hyperplasia in mice with a targeted deletion in the Dutt1/Robo1 gene. Proceedings of the National Academy of Sciences 2001; 98: 15062-15066.

34 Gremlich S, Roth-Kleiner M, Equey L, Fytianos K, Schittny JC, Cremona TP. Tenascin-C inactivation impacts lung structure and function beyond lung development. Sci Rep 2020; 10: 5118.

35 Li Y, Liang J, Yang T, Monterrosa Mena J, Huan C, Xie T et al. Hyaluronan synthase 2 regulates fibroblast senescence in pulmonary fibrosis. Matrix Biology 2016; 55: 35-48.

36 Domingo-Gonzalez R, Zanini F, Che X, Liu M, Jones RC, Swift MA et al. Diverse homeostatic and immunomodulatory roles of immune cells in the developing mouse lung at single cell resolution. Elife 2020; 9: e56890.

$37 \mathrm{Xu} \mathrm{J}$, Yu H, Sun X. Less Is More: Rare Pulmonary Neuroendocrine Cells Function as Critical Sensors in Lung. Dev Cell 2020; 55: 123-132.

38 Degan S, Lopez GY, Kevill K, Sunday ME. Gastrin-Releasing Peptide, Immune Responses, and Lung Disease. Annals of the New York Academy of Sciences 2008; 1144: $136-147$.

39 Kho AT, Chhabra D, Sharma S, Qiu W, Carey VJ, Gaedigk R et al. Age, Sexual Dimorphism, and Disease Associations in the Developing Human Fetal Lung Transcriptome. Am J Respir Cell Mol Biol 2016; 54: 814-821.

40 Clair G, Bramer LM, Misra R, McGraw MD, Bhattacharya S, Kitzmiller JA et al. Proteomic Analysis of Human Lung Development. Am J Respir Crit Care Med 2021. doi:10.1164/rccm.202008-33030C.

41 Du Y, Clair GC, Al Alam D, Danopoulos S, Schnell D, Kitzmiller JA et al. Integration of transcriptomic and proteomic data identifies biological functions in cell populations from human infant lung. Am J Physiol Lung Cell Mol Physiol 2019; 317: L347-L360. 
a

Sample Number of

b

(weeks) Number

14+1 bioRxiv preprint doi: https

$14+3$ (which was not certifieg

\begin{tabular}{|l|l|}
\hline $15+1$ & 3568 \\
\hline $15+5$ & 2685 \\
\hline $16+0$ & 1747 \\
\hline $16+3$ & 1150 \\
\hline $17+5$ & 2014 \\
\hline $18+2$ & 4848 \\
\hline $19+0$ & 1859 \\
\hline
\end{tabular}

$\sum_{\supset}^{5}-5$

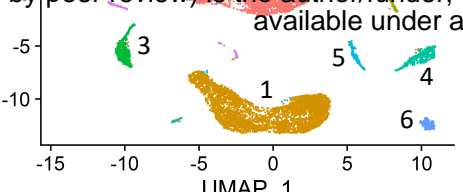

0: Stroma 1

1: Distal airway epithelium

- 2: Stroma 2

- 3: Pericytes

4: Vascular endothelium
C ${ }^{100}$ 管 this version posted December 23, 2021. Whe copyr

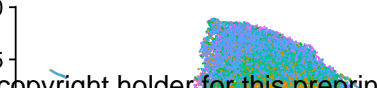
theopreprint in perpetuity. It is made

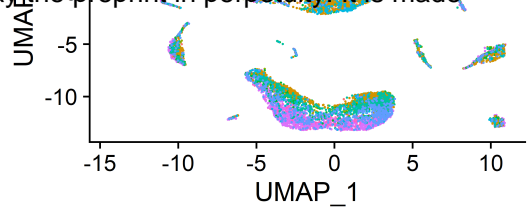

6: Lymphatic endothelium

7: Ciliated cells

- $14+1 \bullet 15+5 \cdot 17+5$

$14+3 \cdot 16+0 \cdot 18+2$

- $15+1 \cdot 16+3 \cdot 19+0$
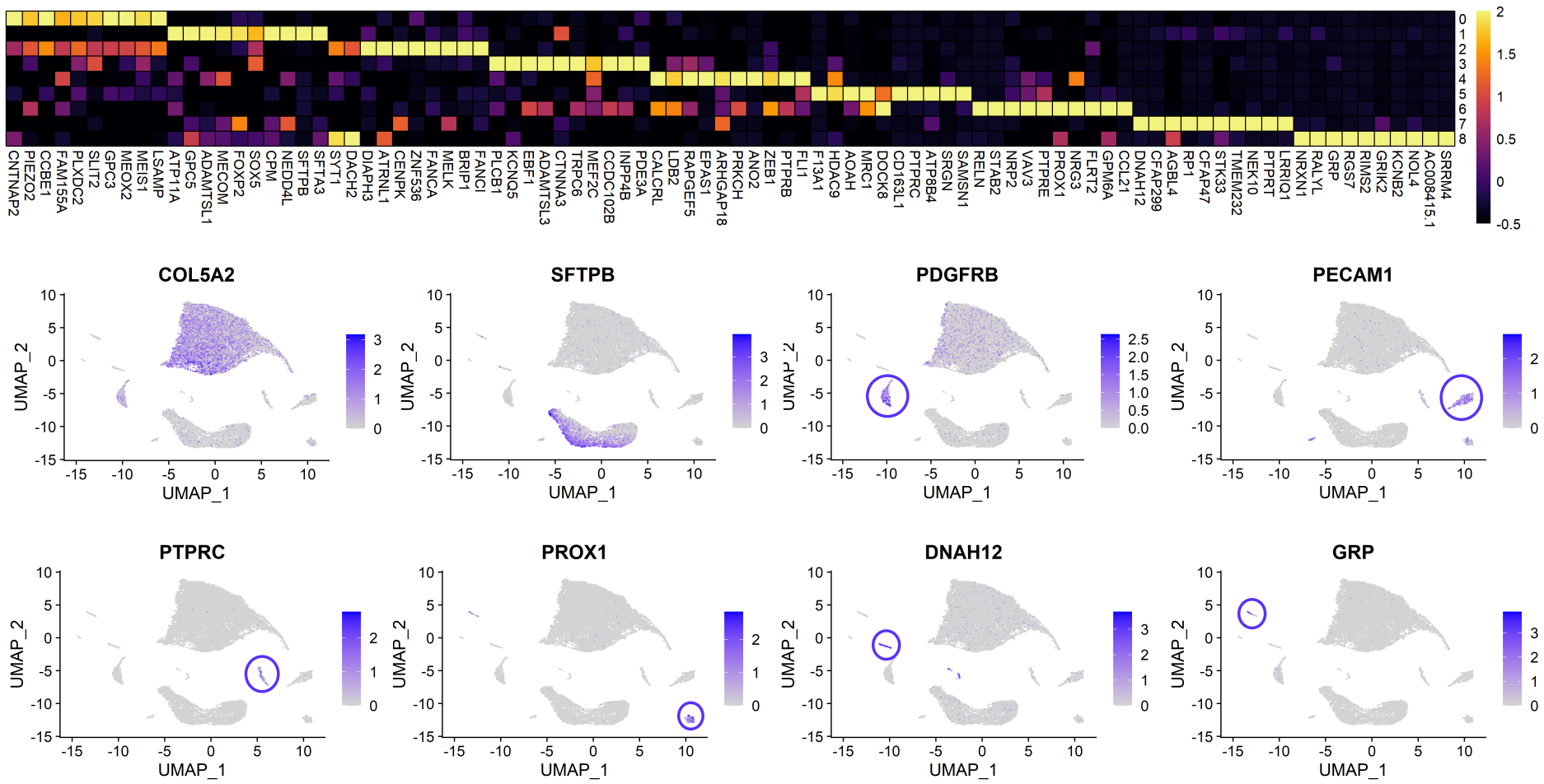


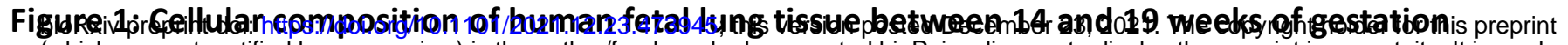

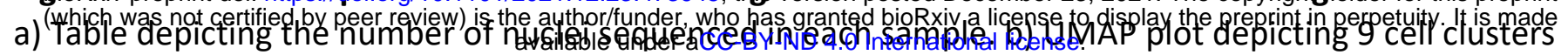
identified by snRNA-seq analysis of fetal lung tissue collected between 14 and 19 weeks of gestation. c) Bar graph depicting the percentual contribution of individual cell populations at different developmental stages. d) UMAP plot depicting the distribution of fetal lung cells based on developmental age. e) Heatmap of top ten most differentially expressed genes across fetal lung cell clusters depicted in panel $b$. f) UMAP plots showing expression levels for canonical markers of lung cell populations, including stroma (COL5A2), distal airway epithelium (SFTPB), pericytes (PDGFRB), vascular endothelium (PECAM1), immune cells (PTPRC), lymphatic endothelium (PROX1), ciliated cells (DNAH12) and pulmonary neuroendocrine cells (GRP). The intensity of expression is indicated by purple coloring. Expression values in heatmaps and UMAP plots represent Z-score-transformed $\log (T P 10 k+1)$ values. Log(TP10k+1) corresponds to logtransformed UMIs per 10k. 


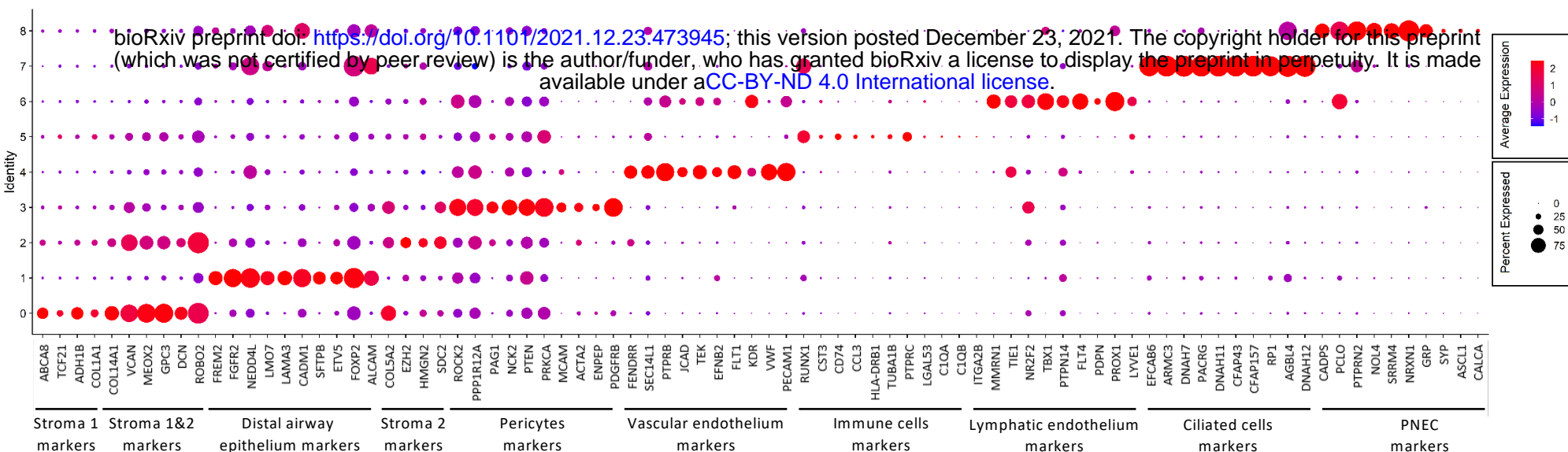

b

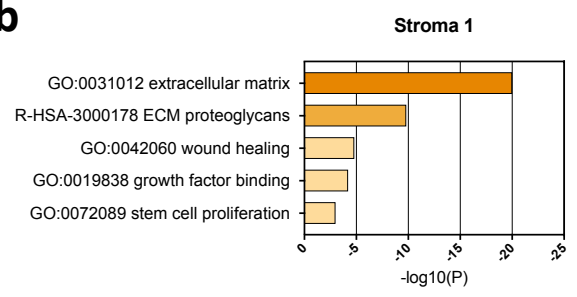

Stroma 2

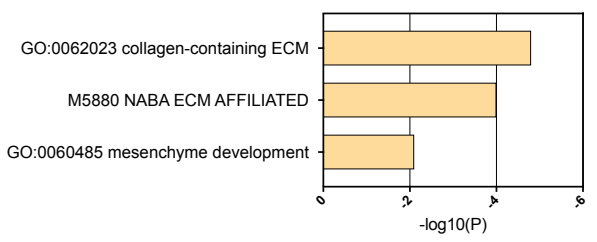

Distal airway epithelium

R-HSA-3000178 ECM proteoglycans GO:0005912 adherens junction GO:0016324 apical plasma membrane GO:0016323 basolateral plasma membrane GO:0007585 respiratory gaseous exchange

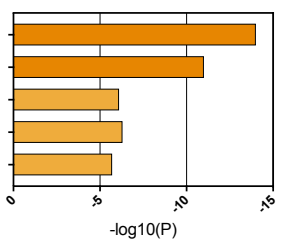

Pericytes markers markers

markers

PNEC

Lymphatic endothelium

Pericytes

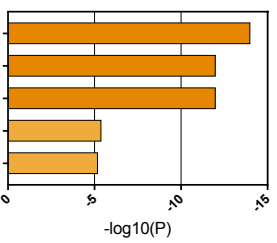

Vascular endothelium

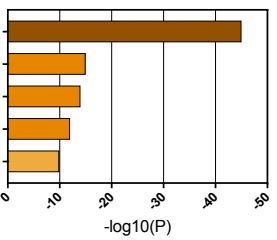

Immune cells

R-HSA-512988 IL-3, IL-5 and GM-CSF signaling R-HSA-449147 Signaling by Interleukins GO:0030099 myeloid cell differentiation R-HSA-9607240 FLT3 Signaling WP3929 Chemokine signaling pathway

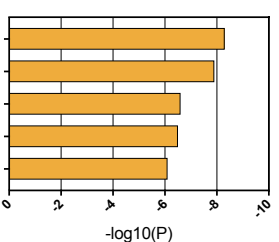

GO:0001944 vasculature development GO:0003013 circulatory system process GO:0060840 artery development GO:0001945 lymph vessel developmen GO:0003158 endothelium developmen

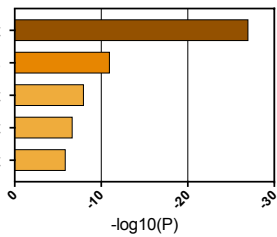

Ciliated cells

GO:0005929 cilium GO:0031514 motile cilium GO:0044447 axoneme part GO:0070286 axonemal dynein complex assembly GO:0036064 ciliary basal body

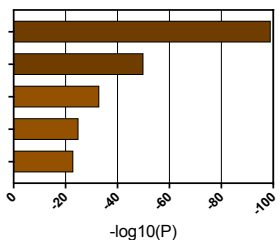

Pulmonary neuroendocrine cells

GO:0099537 trans-synaptic signaling GO:0098794 postsynapse GO:0030424 axon GO:0023061 signal release GO:0098793 presynapse

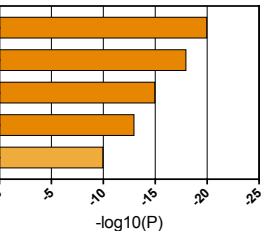




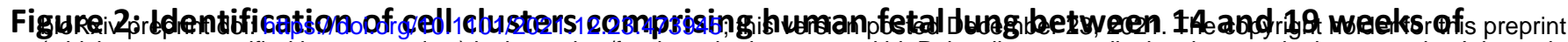
gestiatio was not certified by peer review) is the authorffunder, who has granted bioRxiv a license to display the preprint in perpetuity. It is made a) Dotplot depicting the expression of most commonly used markers for each cell cluster as described in literature. Expression levels in the dotplot are presented as $\log ($ TP10k+1) values. $\log (T P 10 k+1)$

corresponds to log-transformed UMIs per 10k. b) Selected regulated pathways for each cluster identified by gene set enrichment analysis. All terms are significantly enriched (adjusted $p$-value $<0.05$ ). Plotted values represent -log10(p-value). 


\begin{tabular}{cc}
\hline $\begin{array}{c}\text { Sample } \\
\text { (weeks) }\end{array}$ & $\begin{array}{c}\text { Number } \\
\text { of nuclei }\end{array}$ \\
\hline $14+1$ & 1111
\end{tabular}

b

c

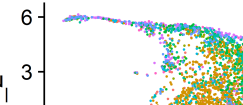

d

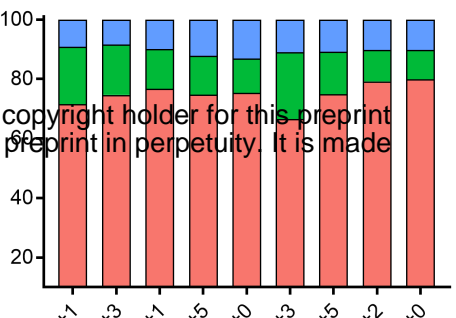

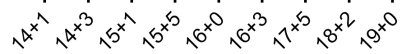

Stromal subclusters

\begin{tabular}{cc}
$14+($ which was & w \\
$15+1$ & 2792 \\
$15+5$ & 1862 \\
$16+0$ & 1139 \\
$16+3$ & 788 \\
$17+5$ & 1372 \\
$18+2$ & 3358 \\
$19+0$ & 959 \\
\hline
\end{tabular}

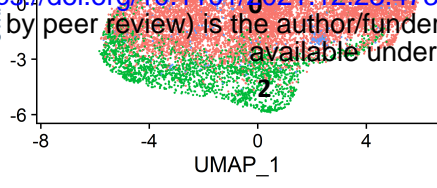

Stromal subclusters

$0 \quad 1$

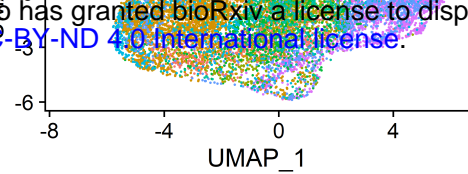

- $14+1 \bullet 15+5 \bullet 17+5$

- $14+3 \cdot 16+0 \cdot 18+2$

$\begin{array}{lll}15+1 & 16+3 \cdot 19+0\end{array}$

e
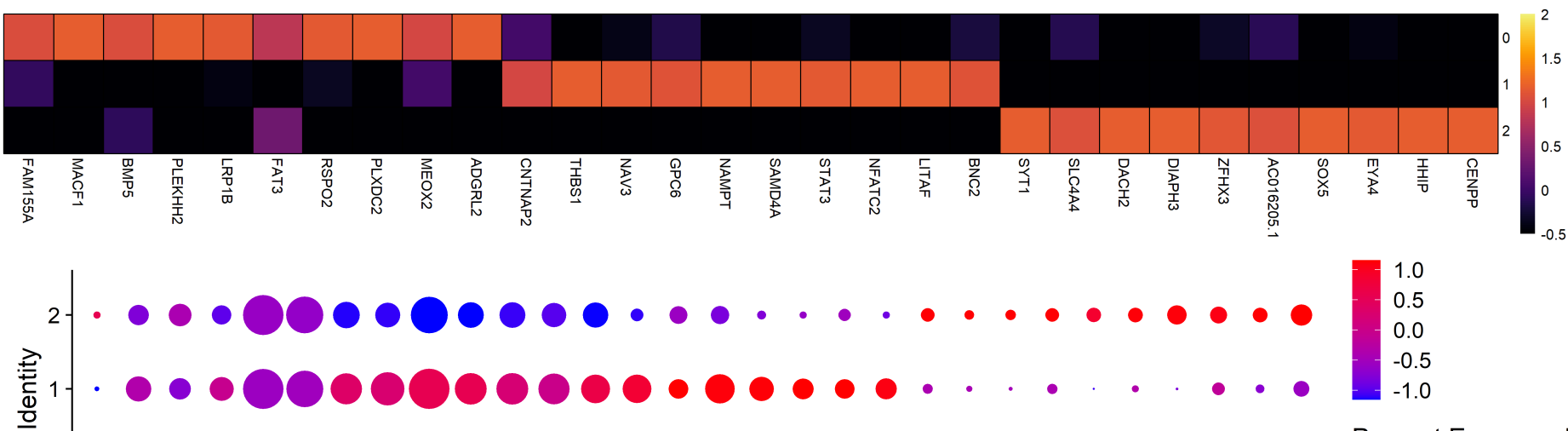

- 10000000000 Percent Expressed

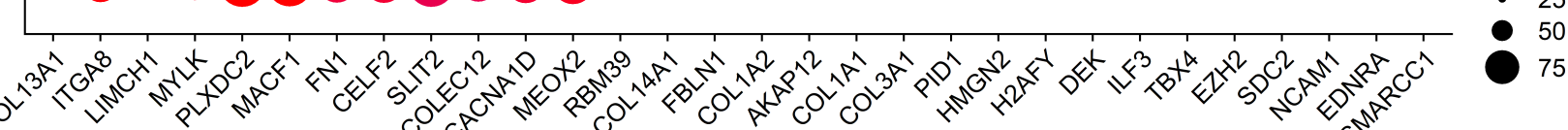

COL13A1+ matrix

fibroblast markers
General matrix

fibroblast markers
COL14A1+ matrix

fibroblast markers
Mesenchymal progenitor

markers g
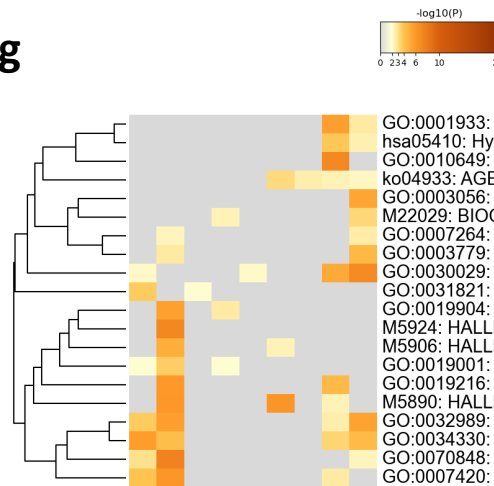

GO:0001933: negative regulation of protein phosphorylation

hsa05410: Hypertrophic cardiomyopathy (HCM) GO:0010649: regulation of cell communication by electrical coupling
ko04933: AGE-RAGE signaling pathway in diabetic complications GO:0003056: regulation of vascular associated smooth muscle contraction M22029: BIOCARTA PLC PATHWAY

GO:0007264: small GTPase mediated signal transduction GO:0003779: actin binding

GO:0030029: actin filament-based process GO:0019904: protein domain specific binding M5924: HALLMARK MTORC1 SIGNALING GO:0019001: guanyl hucleotide binding GO:0019216: regulation of lipid metabolic process GO:0032989: cellular component morphogenesis GO:0034330: cell junction organization GO:000848: response to growth factor

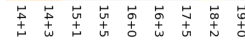

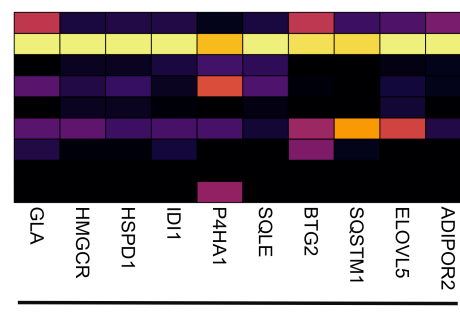

MTORC1 signaling
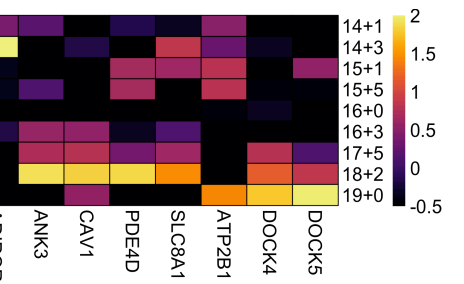

Regulation of cell communication by electrical

Regulation of vascular associated coupling h

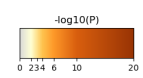

0019904 protein domain specific binding GO: 19003706 : regulation of hemopoiesis GO:0003012: muscle system process

tion development muscle structure development GO:0030198: extracellular matrix organization

M231: PID KIT PATHWAY
GO:2001237: negative regulation of extrinsic apoptotic signaling pathway

GO:0061588: calcium activated phospholipid scrambling GO:0021813: cell-cell adhesion involved in neuronal-glial interactions GO:0030155: regulation of cell adhesion mitotic spind e microtubules to kinetochore GO:0006024: glycosaminoglycan biosynthetic process M5942: : HALLMARK UV RESPONSE DN M5890: HALLMARK TNFA SIGNALING VIA

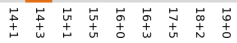

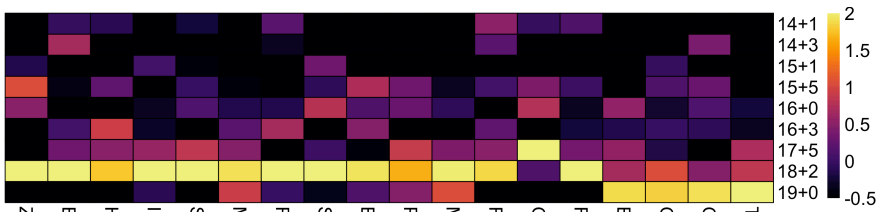




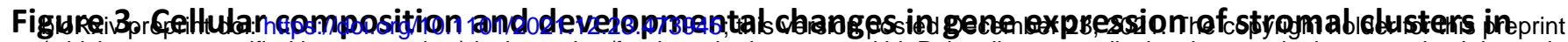

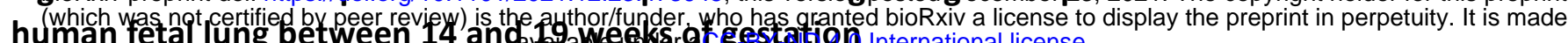
a) Table depicting the number of stromal cells nuclei sequenced in each sample. b) UMAP plot depicting the 3 stromal subclusters identified within the fetal lung stroma. c) UMAP plot depicting the distribution of fetal lung stromal cells based on the developmental age. d) Percentual contribution of the different lung stromal subclusters in each individual sample. e) Heatmap depicting the top ten most differentially expressed genes across fetal lung stromal subclusters shown in panel b). f) Dotplot depicting the expression of stromal markers known to be associated with $\mathrm{COL} 13 A 1^{+}$matrix fibroblasts (subcluster 0 ), COL14A1 $1^{+}$matrix fibroblasts (subcluster 1 ), general matrix fibroblasts (subcluster 0 and 1 ), and mesenchymal progenitors (subcluster 2 ) in stromal subclusters. g) Heatmaps depicting the enriched terms associated with individual GAs as identified by multi-list enrichment analysis based on DSA in the main cluster stroma 1 (top), and heatmap depicting the expression level of genes associated with selected enriched terms (bottom). h) Heatmaps depicting the enriched terms associated with individual GAs as identified by multi-list enrichment analysis based on DSA in the main cluster stroma 2 (top), and heatmap depicting the expression level of genes associated with selected enriched terms (bottom). Expression levels in the heatmaps and dotplots are presented as $\log (T P 10 k+1)$ values. $\log (T P 10 k+1)$ corresponds to log-transformed UMIs per 10k. 


\begin{tabular}{cc}
\hline $\begin{array}{c}\text { Sample } \\
\text { (weeks) }\end{array}$ & $\begin{array}{c}\text { Number of } \\
\text { nuclei }\end{array}$ \\
\hline $14+1$ & 37 \\
\hline $14+3$ & 522 \\
\hline $15+1$ & 378 \\
$15+5$ & 502 \\
\hline $16+0$ & 467 \\
\hline $16+3$ & 236 \\
\hline $17+5$ & 491 \\
\hline $18+2$ & 757 \\
\hline $19+0$ & 712 \\
\hline
\end{tabular}

b

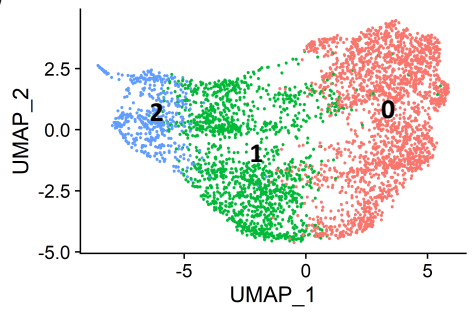

Distal airway epithelial subclusters
C

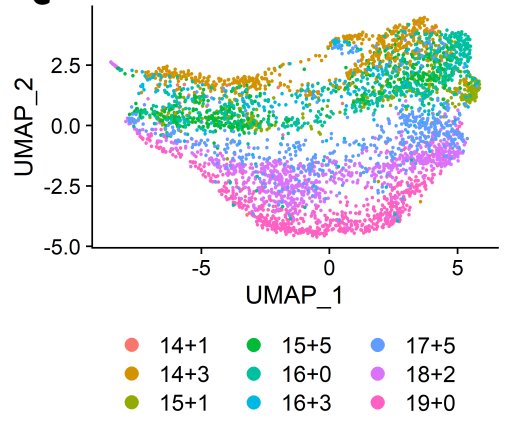

d

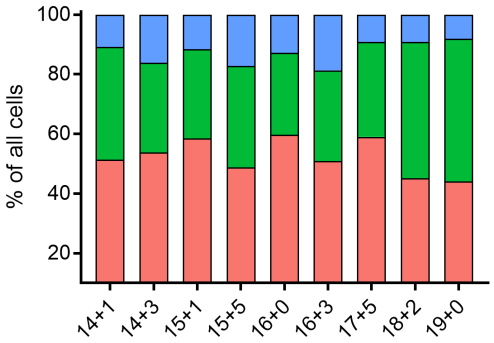

Distal airway epithelial subclusters

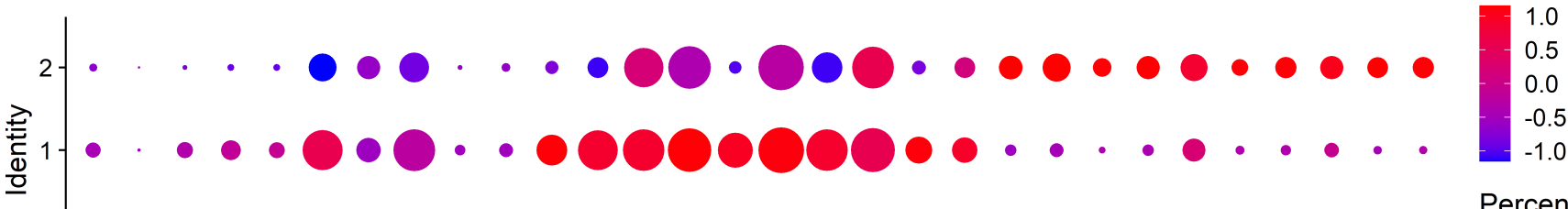

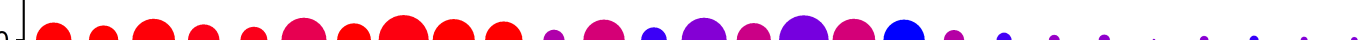

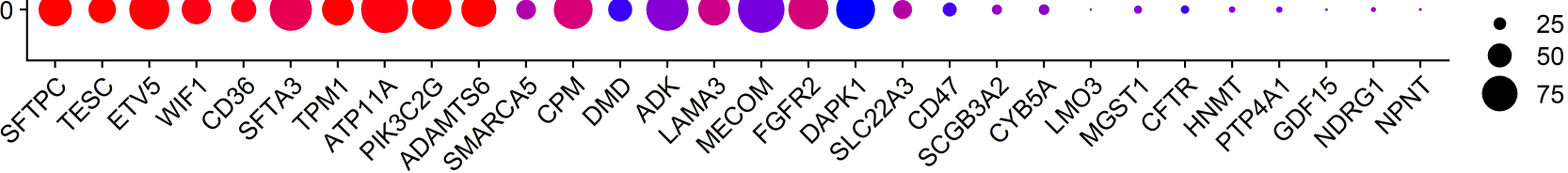

Bud tip progenitor markers

Bud tip adjacent markers

Secretory progenitor markers

g
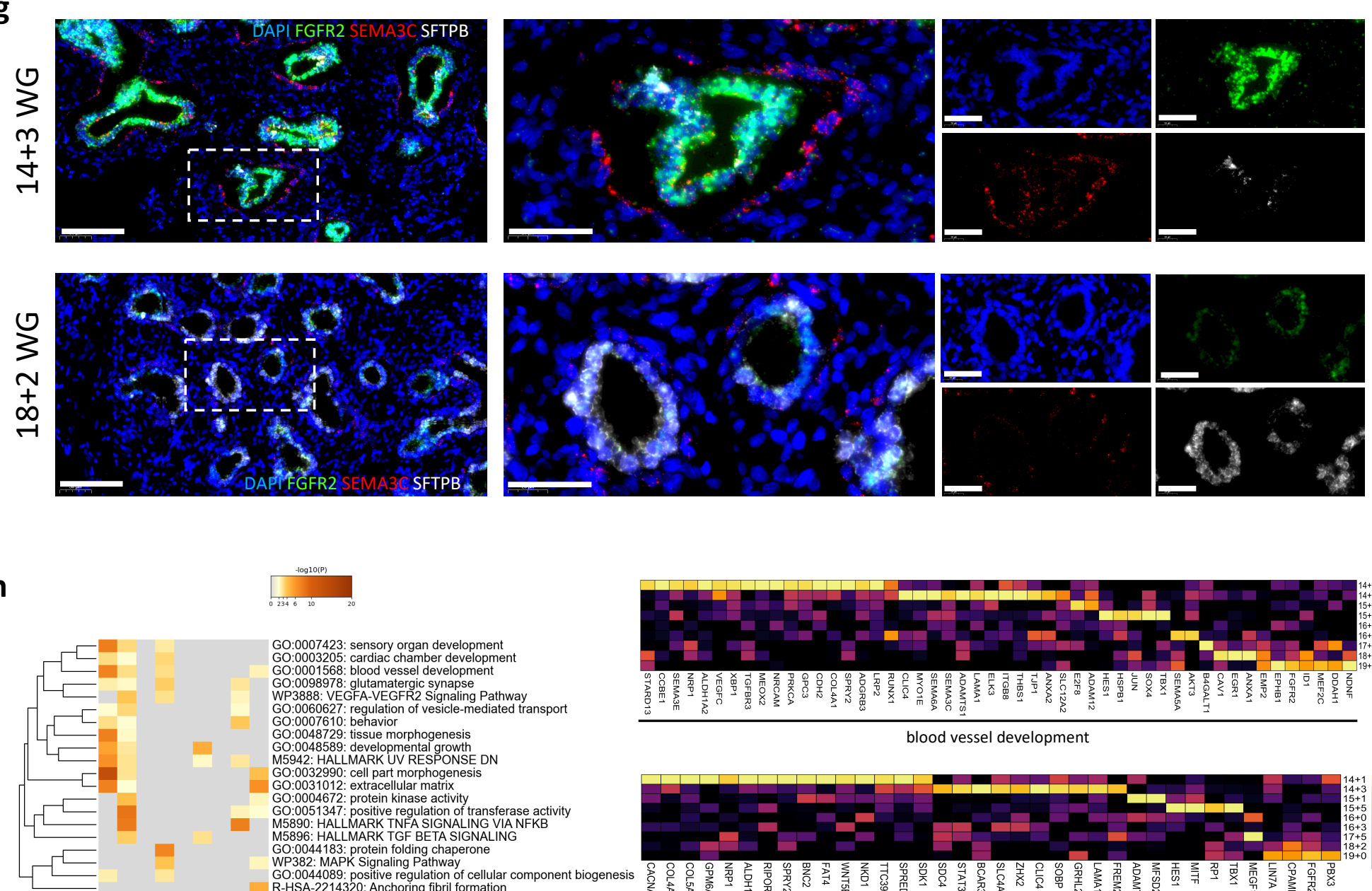

GO:0007423: sensory organ development GO:0001568:

GO:0098978: glutamatergic synapse

GO:0060627: regulation of vesicle-mediated transport

GO:0007610: behavior

5942: HALLMARK UV RESPONSE DN

GO:0032990: cell part morphogenesis

GO:0031012: extracellular matrix

0.0051347: positive regulation of transferase activity

M5890: HALLMARK TNFA SIGNALING VIA NFKB

GO:00044183: protein folding chaperone

GP382: MAPK Signaling Pathway
GO:0044089: positive regulation of cellular
R-HSA-2214320: Anchoring fibril formatio

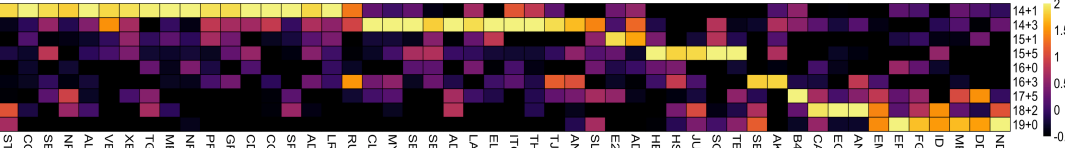

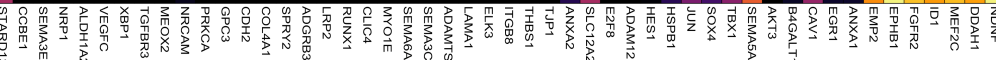

blood vessel development

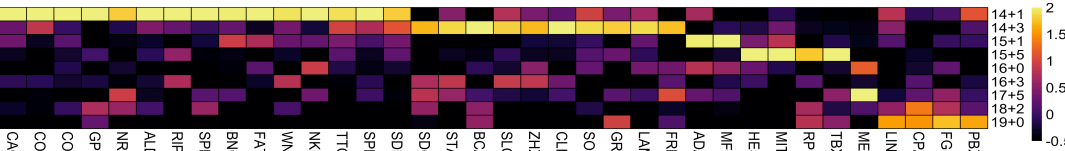


Figure 4. Cellular composition, developmental changes in gene expression, and spatial localization of distal airway epithelial cells in human fetal lung between 14 and 19 weeks of gestation

a) Table depicting the number of distal airway epithelial cells nuclei sequenced per sample. b) UMAP plot depicting the 3 epithelial subclusters identified within the distal airway epithelium cluster; c) UMAP plot depicting the distribution of fetal lung distal airway epithelium cells based on developmental age. d) Percentage contribution to the different lung epithelial subclusters per sample. e) Heatmap depicting the top ten most differentially expressed genes across fetal lung epithelial subclusters shown in panel b). f) Dotplot depicting the expression of epithelial markers known to be associated with bud tip progenitor cells (subcluster 0 ), bud tip adjacent cells (subcluster 1 ) and secretory progenitors (subcluster 2 ). g) Representative fluorescent RNA in situ hybridization pictures showing co-expression of FGFR2 (green), SEMA3C (red) and SFTPB (white) in the airway epithelium in human fetal lungs at $14+3$ (top) and 18+2 (bottom) weeks of gestation. Magnification at x20 (scale bar $100 \mu \mathrm{m}$ ) and x63 (scale bar $40 \mu \mathrm{m}$ ). Expression levels in the heatmaps and dotplots are presented as $\log ($ TP10k+1) values. $\log ($ TP10k+1) corresponds to log-transformed UMIs per 10k. h) Heatmaps depicting the enriched terms associated with individual GAs as identified by multi-list enrichment analysis based on DSA in the main cluster distal airway epithelium (left), and heatmap depicting the expression level of genes associated with selected enriched terms (right). 


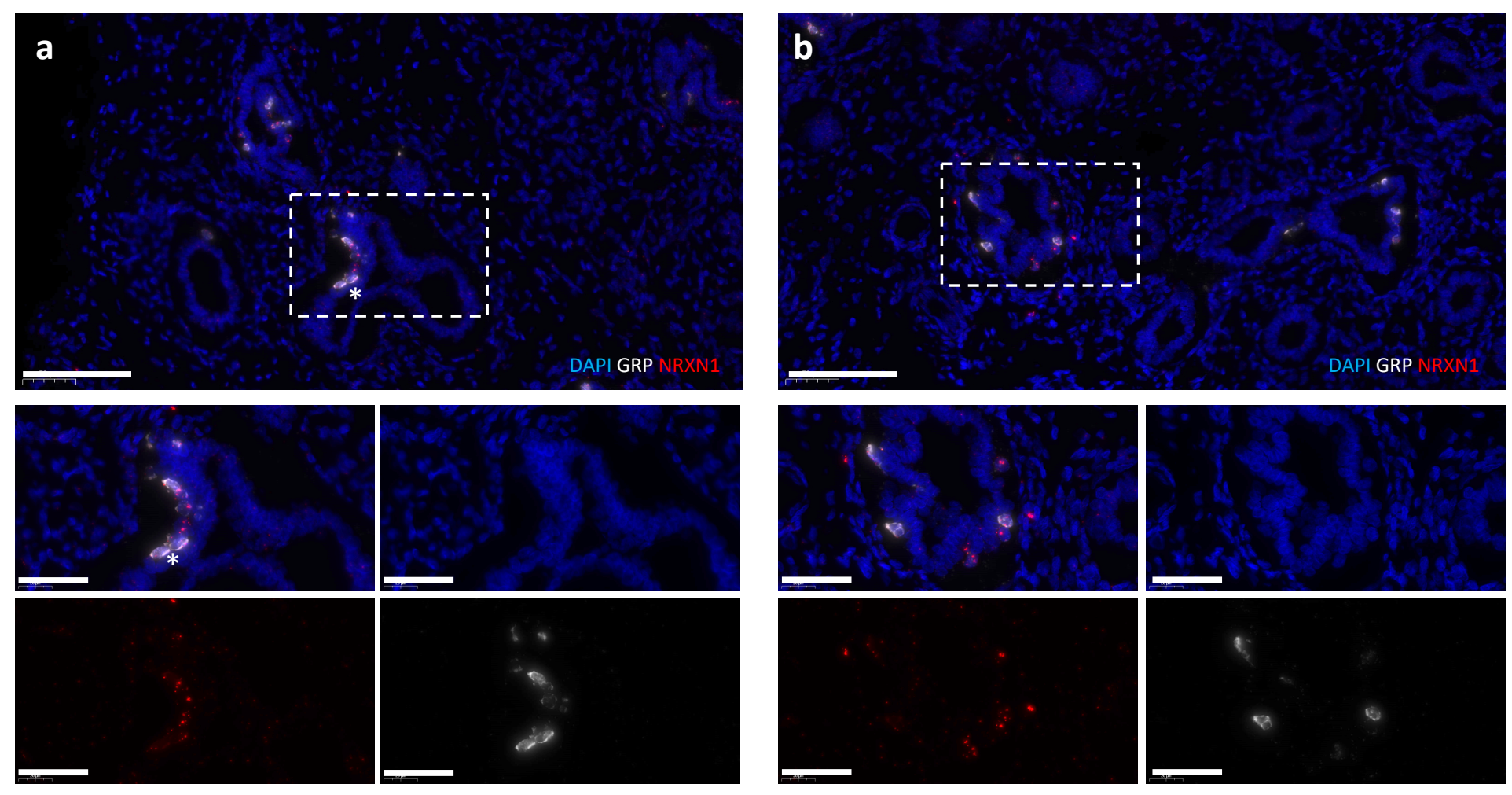


Figure 5. Fluorescent RNA in situ hybridization of pulmonary neuroendocrine cells in the human fetal lung

Representative fluorescent RNA in situ hybridization pictures showing co-expression of NRXN1 (red) and GRP (white) in PNEC in human fetal lungs at a) $14+3$ and b) $15+5$ weeks of gestation. Both, isolated $N R X N 1^{+} / G R P^{+}$PNECs and small PNECs clusters (neuroendocrine bodies, white ${ }^{*}$ ) were noted. Magnification at x20 (top, scale bar $100 \mu \mathrm{m}$ ) and x63 (bottom, scale bar $40 \mu \mathrm{m}$ ). 
a

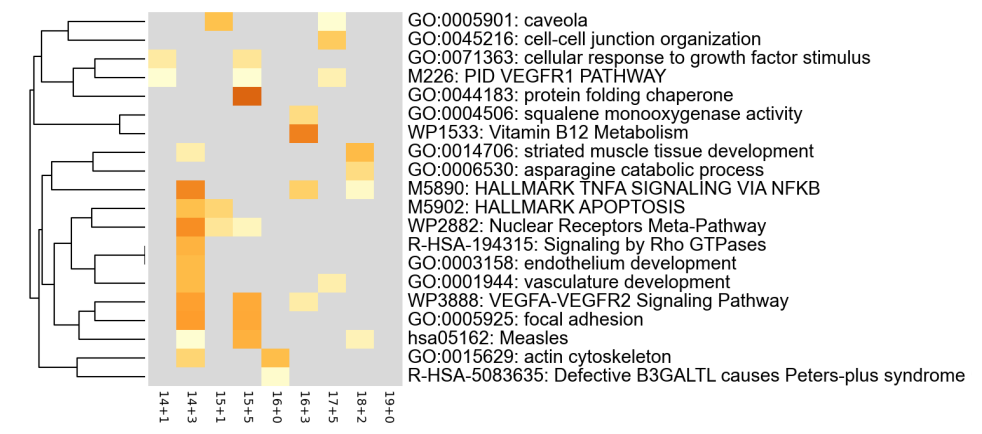

b
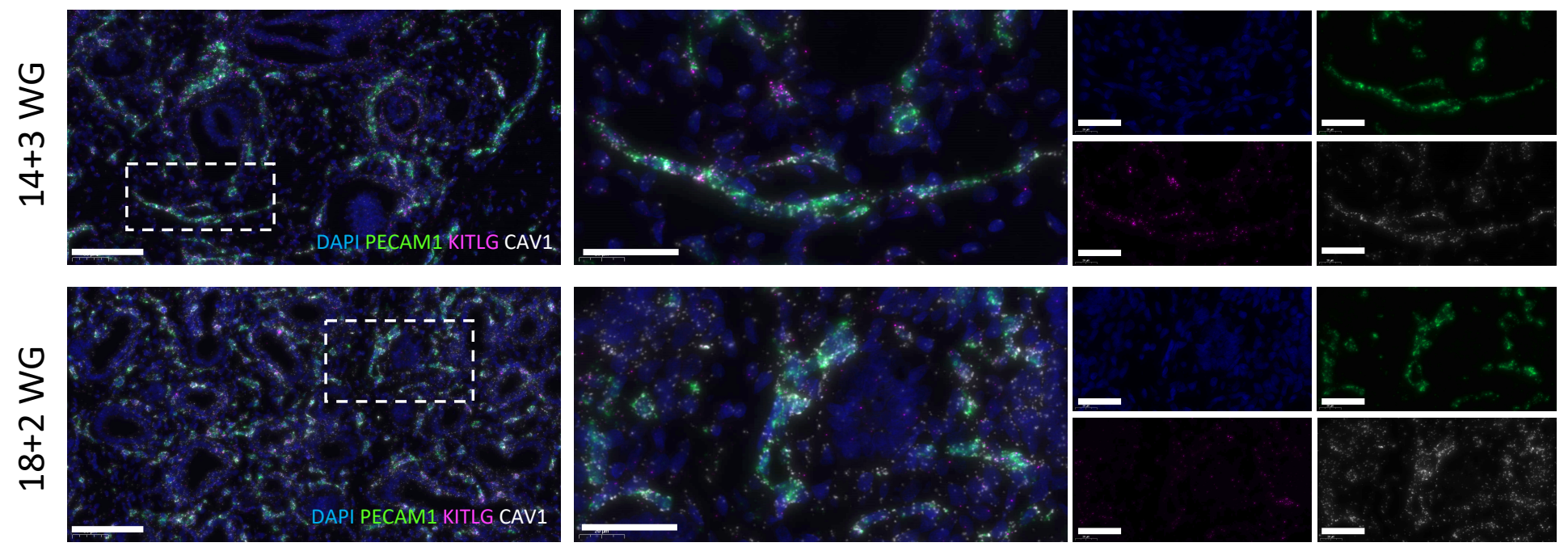
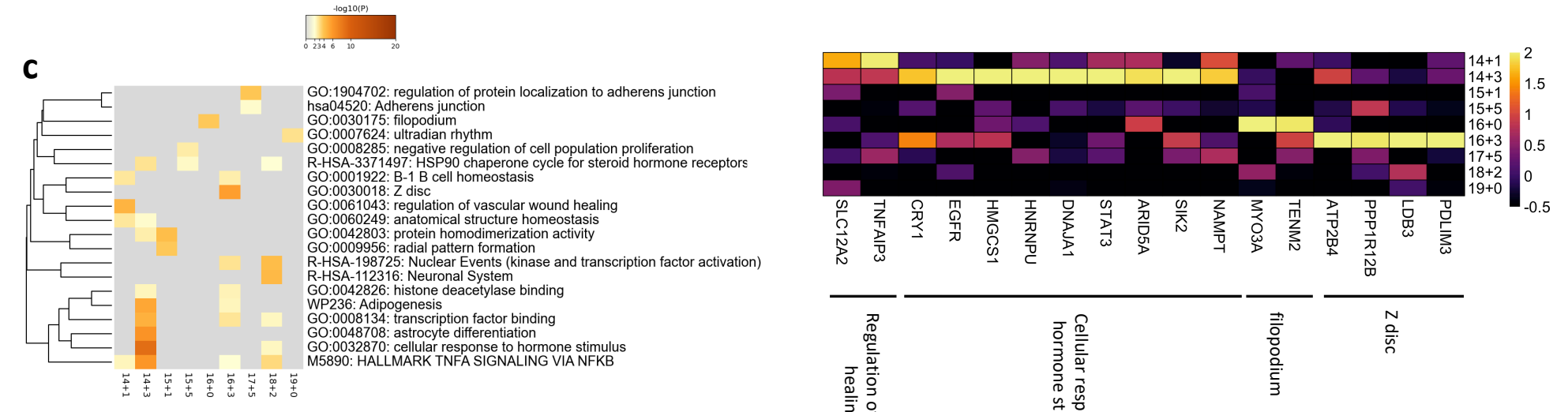

d
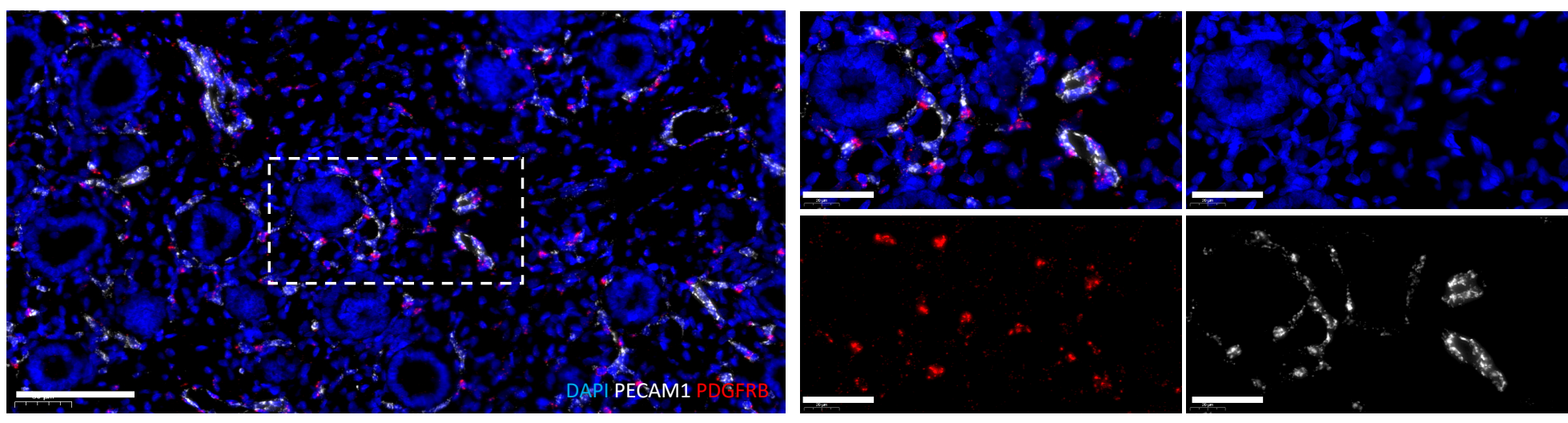
Figure 6. Developmental changes in gene expression and spatial localization of vascular endothelium and pericytes

a) Heatmaps depicting the enriched terms associated with individual GAs as identified by multi-list enrichment analysis based on DSA in the main cluster vascular endothelium (left), and heatmap depicting the expression level of genes associated with selected enriched terms (right). b) Representative fluorescent RNA in situ hybridization pictures showing co-expression of PECAM1 (green), KITLG (magenta) and CAV1 (white) in the vascular endothelium in human fetal lungs at $14+3$ (top) and 18+2 (bottom) weeks of gestation. Magnification at x20 (scale bar $100 \mu \mathrm{m}$ ) and x63 (scale bar $40 \mu \mathrm{m})$. c) Heatmaps depicting the enriched terms associated with individual GAs as identified by multi-list enrichment analysis based on DSA in the main cluster pericytes (left), and heatmap depicting the expression level of genes associated with selected enriched terms (right). Expression levels in the heatmaps are presented as $\log (T P 10 k+1)$ values. Log(TP10k+1) corresponds to log-transformed UMIs per 10k. d) Representative fluorescent RNA in situ hybridization pictures showing co-localization of PECAM1+ endothelial cells (white) and PDGFRB+ pericytes (red) in human fetal lungs at $18+2$ weeks of gestation. Magnification at x20 (scale bar $100 \mu \mathrm{m}$ ) and $\times 63$ (scale bar $40 \mu \mathrm{m}$ ). 


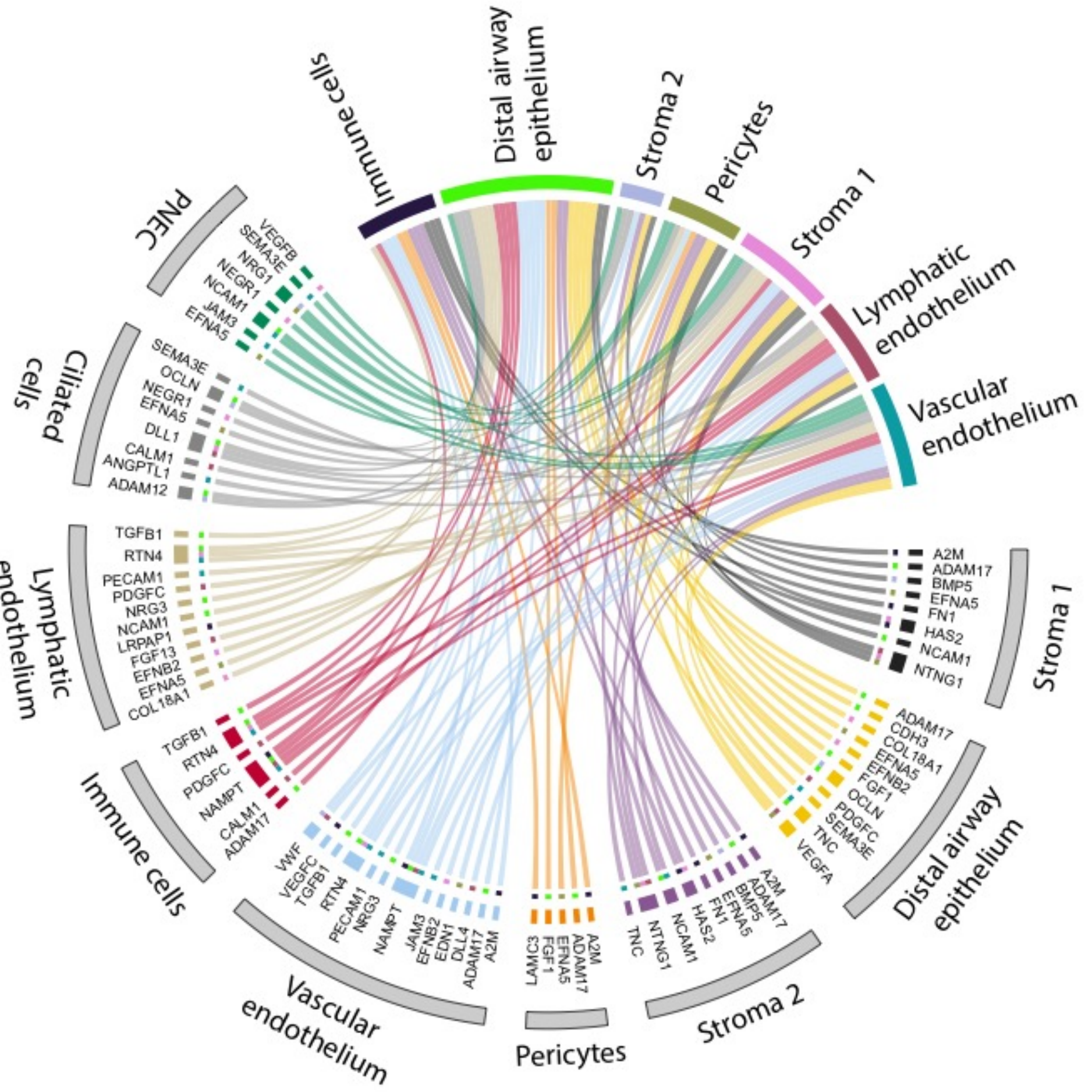


Figure 7: Cell communication pathways induced over time

Circos plot showing inferred cell communications identified with NicheNet by comparing the two latest GA $(18+2$ and $19+0$ weeks) with the two earliest GA $(14+1$ and $14+3$ weeks). Colored populations in the upper part of the plot represent the receiving cell populations, while grey-colored populations in the lower part of the plot represent the signal senders and their ligands. Each ligand is connected to the respective receiving population. 

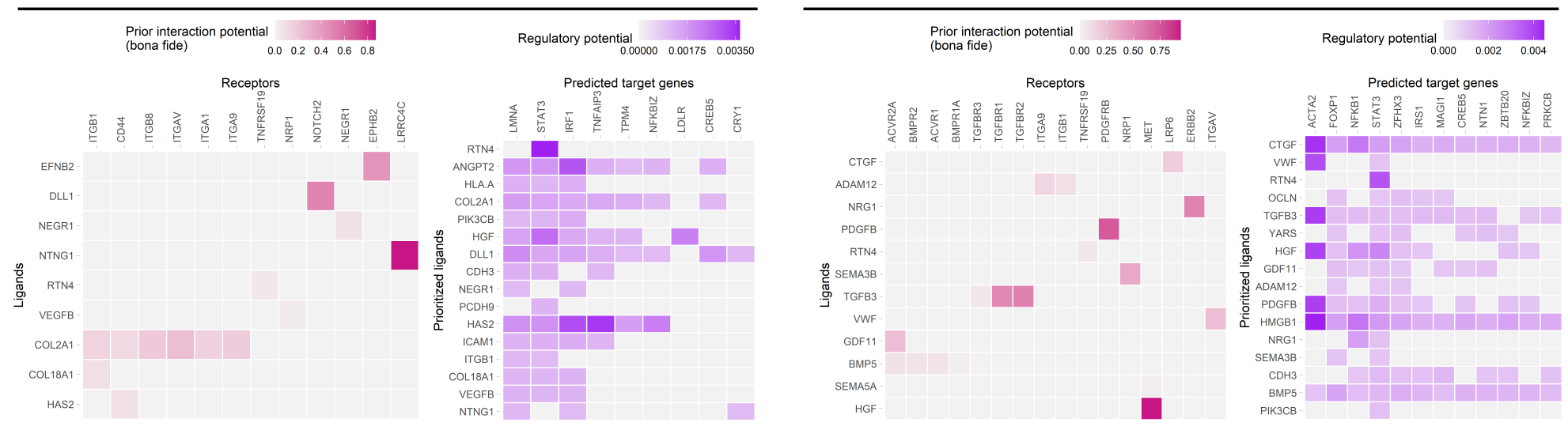

Distal airway epithelium

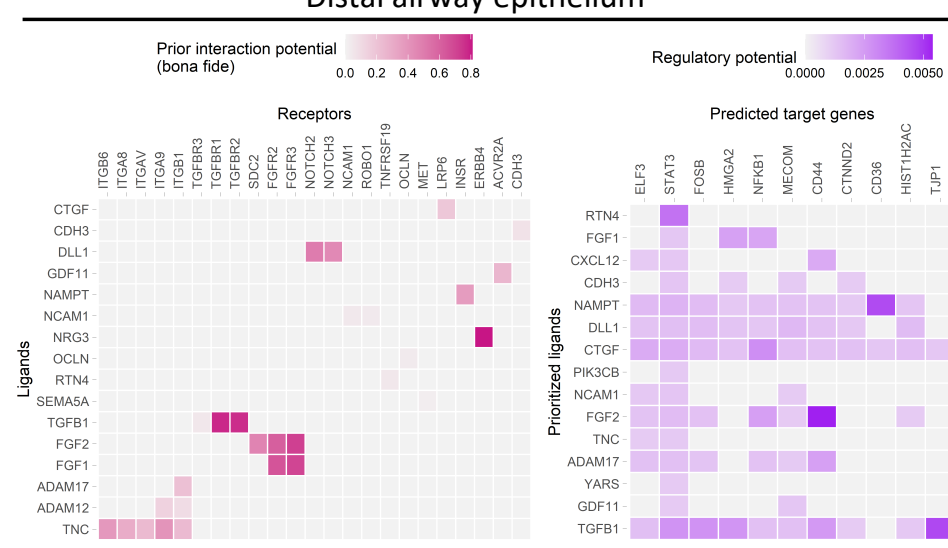

Lymphatic endothelium
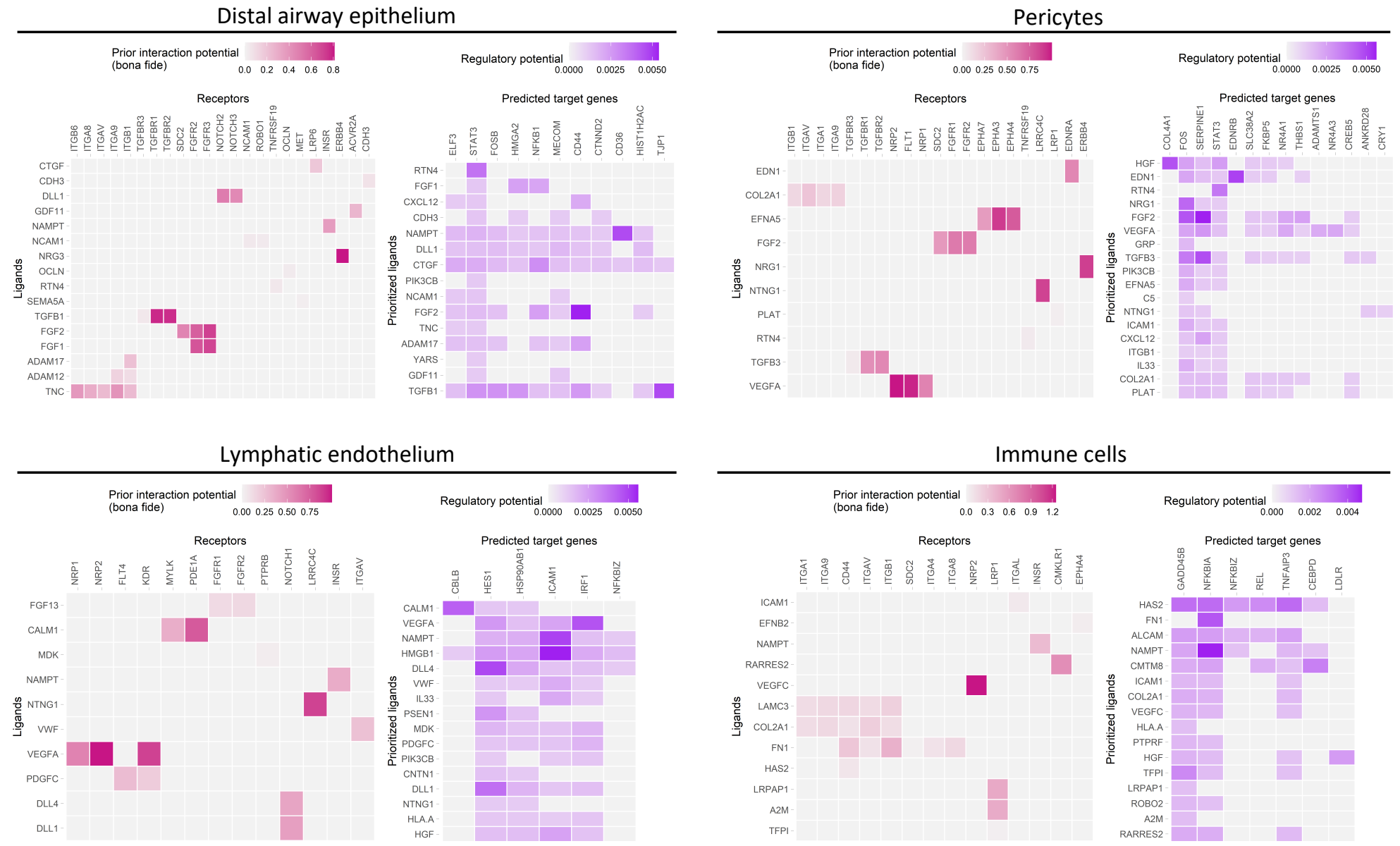

Vascular endothelium

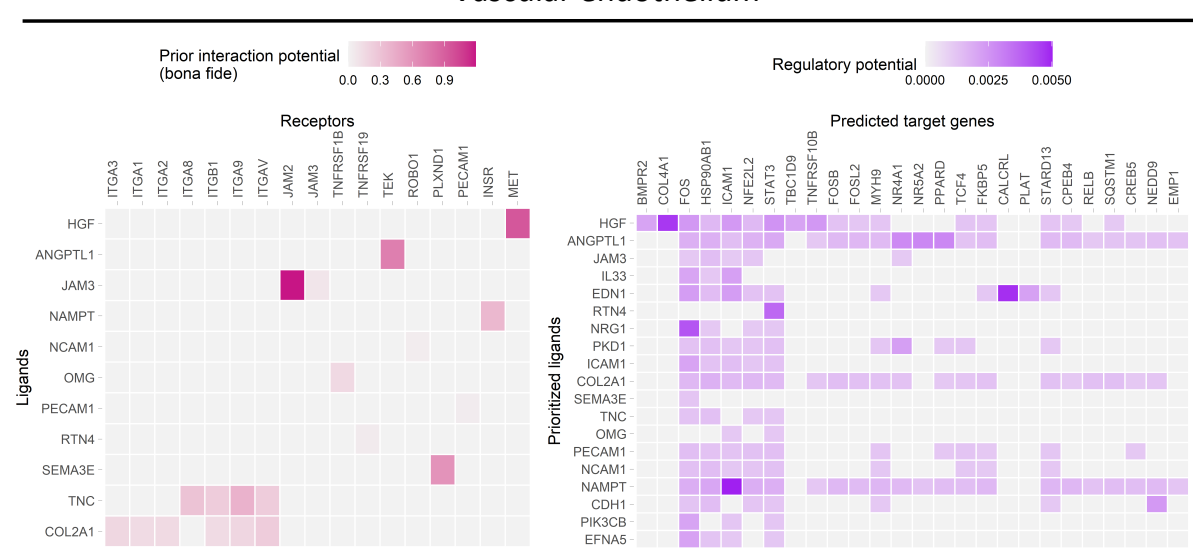


Figure 8. Identified receptors and predicted target genes involved in cell communication

Heatmaps showing bona-fide receptors and predicted target genes for each receiving cell population identified with NicheNet by comparing the two latest GA (18+2 and 19+0 weeks) with the two earliest GA (14+1 and 14+3 weeks). Receiving cell populations include stroma 1 and 2, pericytes, Lymphatic endothelium, distal airway epithelium, immune cells and vascular endothelium. For each cell population, pink heatmap reports bona fide receptors and their ligands and purple heatmap shows predicted target genes according to prioritized ligands. 